\title{
Blockchain a Challenge for the New Future Mobile Economy
}

\author{
Vasile MAZILESCU ${ }^{\star}$
}

\begin{tabular}{l}
\hline \multicolumn{1}{c}{ A R T I C L E I N F O } \\
\hline Article history: \\
Accepted March 2019 \\
Available online April 2019 \\
\hline JEL Classification \\
M10 \\
Keywords: \\
Blockchain, Business processes, \\
Security-based decentralization
\end{tabular}

\begin{abstract}
A B S T R A C T
This paper analyzes the development and implementation of blockchain technology as a future basis for transforming models, roles and business processes in the context of the new mobile economy. Transparency of transactions must be a common desideratum of all companies involved in the development of blockchain platforms, because only such an approach can enhance widespread adoption. In this respect, close cooperation between companies developing this technology and regulatory authorities is needed to increase confidence and create a framework that takes into account the particularities of this technology. Major technology developers, such as IBM, Oracle and Amazon, have been involved in blockchain services. Other companies have begun to test the technology, establishing the first cases of use and prototypes of blockchain-based solutions Organizations can start from a low level, but they need to set a goal for the initiative so that other participants can identify it and then be able to align it. Given the fact that blockchain technology is being properly utilized, it has a significant potential to stimulate the transition to more decentralized, safer solutions with a greater potential to conserve resources and mobilize communities.
\end{abstract}

(c) 2019 EAI. All rights reserved.

\section{Introduction}

Blockchain is the generic term for distributed registry technologies programmed to record or track various forms of value - from financial transactions to personal medical records or personal documents [1]. It can also be defined as a protocol that sets a set of rules (in the form of distributed calculations) that ensure the integrity of the changed data and eliminates the need for a trusted third party [4]. Trust or Trust Technology, also seen as the future of the Internet, an Internet of Values or Web 3.0, especially the current Internet of Information [2]. In this digital register any information entered is encrypted in mathematical formulas and each mathematical formula authenticates and validates another mathematical formula containing other encrypted data in turn [18]. This decentralized data base contains all the information of all transactions involving value and which are encrypted on the blockchain, with the permission to verify their authenticity at any time. Blockchain operates on a decentralized and distributed network with at least two computing units [19]. It is decentralized because the relationships are peer-to-peer and is distributed because the register is not stored in one place, but there is a copy of the entire blockchain at each node, operation performed at the network node input and then updated at closing each block. Smart contracts are basically blockchain programs, not very different from paper contracts. A smart contract can be programmed so that its clauses are automatically fulfilled once both parties have fulfilled their obligations [12]. Being created exclusively in the blockchain, they cannot be modified once they are created, and their good completion by both parties can be validated by all network users. At the moment, the largest blockchain that allows smart deals is Ethereum. Goods manufacturers worldwide can use the blockchain to create digital certificates for each product. This can help consumers, as well as distributors, to immediately detect the origin of a product, regardless of the reason for the request. Through sites, users can enter various documents directly into the blockchain, which can allow them to prove the authenticity of these documents at any time, but also their membership. The ability to record documents in a blockchain can help reduce bureaucracy and ease work in governmental, educational institutions, as well as in healthcare facilities $[5,16]$. Although many attempts have been made to implement an electronic vote, they have not always had the desired results. The most obvious voters' fears are inevitably linked to possible manipulation of the results by hackers or other malicious cybernetic organizations. Blockchain would not only represent a way for votes to be recorded in a database almost impossible to modify, but also a transparent method by which counting of votes can be verified by any system user voter. But as versatile as the blockchain, we must keep in mind that technology is still in its infancy. Blockchain provides a 
minimal protection of its users' personal data, even if transaction history is accessible to anyone. This means that in areas where personal data will be needed (voting, medical records, bank transactions, etc.), their protection will be a challenge [3]. It remains to be seen if the blockchain can be effectively applied in all areas it promises to revolutionize. But even in the unlikely event that the implementation of the blockchain will be a failure, it will certainly serve as inspiration for the future technologies that will depend on day-to-day living in the new mobile economy [17, 20]. Microsoft's involvement in the crypto market began in December 2014 when purchases from Windows and Xbox stores could be paid in Bitcoin. Acceptance of payment in Bitcoin at Microsoft stores came at a time when the adoption of crypto currencies was at a low level. This was the first indication that the company was open to innovation in general and to the crypto market in particular [6]. Microsoft is now developing Blockchain applications and is working on building platforms for companies to develop Blockchain applications such as the Confederal Framework (Coco), a protocol based on Ethereum. For example, Microsoft Azure offers full flexibility in choosing resources, as well as the ability to scale out the characteristics and volumes of these resources in a few clicks, depending on the dynamics of each user's needs. The collaborative approach is how blockchain technology can enhance transparency. This would provide better products for customers and also for the companies involved. The reliable provider today is transparent and its achievement in the field of safety is achieved when responsibility is shared. The five challenges of a blockchain platform are: building an ecosystem, creating a business model, securing data security and access rights, integrating with other platforms, and building a technology system that has performed best in the area of companies. It is important to address all these challenges to ensure that all actors in the mobile economy ecosystem feel involved. The paper is made up of four sections: Introduction, Section 2 analyzes the features of blockchain technology, Section 3 shows the impact of blockchain technology on the mobile economy and some examples of business platforms that are in the development process, and the conclusions are highlighted in Section 4 .

\section{Features of blockchain technology}

Blockchain technology is a decentralized transaction log - in other words, a decentralized transaction platform in a network infrastructure. It can be useful for both transport operators and those working in the logistics sector. Two years ago, the blockchain technology market was estimated at just over two-hundredhundred million dollars. In three years the value should be ten times higher. Applications based on blockchain technology were used in the first phase in the financial industry, data protection, IT, health and energy $[7,8,13]$. Currently, the number of companies in different sectors using this technology is growing rapidly. This technology is increasingly used by carriers and logistics operators as well. Collection of data in the online environment, accounting for individual transactions or log entries in the network and encrypted using cryptographic algorithms. These are just some of the benefits of using blockchain technology. Problems solved by chain-block block technology are the lack of trust in participants who trade resources in the network, but the only point of confidence, being the system itself, decentralization, and thus the elimination of single attack points, the ability to guarantee a reality through the consensus of participants system. Even though technical bases have been set up since the late 1970s, blockchain technology has received increased attention with the emergence of electronic coins, more precisely with the development of Bitcoin. We could summarize that Bitcoin was the first programmable currency. Since 2014, Bitcoin 2.0 has begun to develop, which has involved intelligent contracts and smart assets, decentralized applications (DAPPs) and decentralized autonomous corporations (DACs). One such system as Bitcoin is the product of three components: the blockchain, the protocol, and the coin. The Blockchain is basically a distributed database of records or public information of all transactions or digital events that have been executed and shared between the participating parties. Blockchain can be interpreted as a public "ledger" that contains transaction information. Each transaction in the public register is verified by the consensus of most participants in the system. Once entered, the information cannot be deleted or altered. A hash function (or dispersion function) is mathematical, defined on a set with many elements (possibly infinite) with values in a set with a fixed number. Hash functions are not reversible. A hash function can bind two or more keys of the same hash value. In many applications, it is desirable to minimize the chance of such collisions, which means that the hash function must tie the keys to the hash values as evenly as possible. Although the idea was conceived in the 1950s, the optimal design of hash functions is still an active subject of research and discussion. Hash functions are also used as checksums or cryptographic hash functions, but they should not be confused with verification characters, numeric fingerprints, randomization functions, error correction codes. A hash is what you get with a fixed size after applying a cryptographic functions on a certain content. This result is identified in literature as a hash, digest or even hash. By using hash, integrity of the content can be guaranteed, and in the case of blockchains, they are used to address blocks. Simply, instead of an http address that we are very familiar with, we use a hash. A hash is an alphanumeric sequence of a certain size. The Hash of each block consists of block data plus the hash of the parent block so that if there is a change in a parent block, the hash values of the child blocks will also be changed. A block consists of: Block header and Block body. The header of a block contains the following elements: Block Version (indicates which set of validation registers must follow the block), Merkle Tree Root Hash (the hash value of all transactions in the current block), Timestamp 
(the current universal time in seconds $1 \mathrm{n}$ bits (the target is the threshold below which the hash must be, so the block is valid) nBits is the encoded form of the target threshold, Nonce (a 4-byte field that usually starts with 0 and increases for each calculation has), Parent Block Hash (a 256-bit hash that indicates the previous block). The key features of a blockchain are Decentralization, Persistence and Anonymity. In conventional transaction systems, each transaction must be validated through an agent $[9,11]$.

In essence, the blockchain is an autonomously distributed system that no longer allows for deception. For example, the software on the Ethereum platform (running the Crypto-Coin Ether) allows for the registration in chain blocks of program codes called "smart contracts" that could replace everything that means a mediation service between a buyer and a seller, because they can guarantee the authenticity of a product. As we have shown, in a blockchain, the entire history of all information and transactions is preserved. Virtual coins (crypto currencies) are generated by free software (open source) installed on the computer. Each crypto currency has its own software, whether it's Bitcoin, Ether, Monero, etc. Computers or agents running these software become nodes in a global network, but not any PC or laptop for personal use qualifies for this activity. Agents use computers called ASICs (Application-Specific Integrated Circuit), equipped with specialized hardware to solve the so-called hash, which consumes enormously much electricity. A hash function processes the input and turns it into a hash - a random string of fixed size that will forever be the "mask" of the initial information. Each hash is an identifier of the entire history spent in it in the chain of network entries. Each link in this chain is called a block and is linked and derived from the previous block. The most recent hash encodes all of the existing block chain memory in the ASIC world network. Once the software is installed, an ASIC becomes a "miner", meaning it starts to generate new blocks, depending on the speed with which the hash function resolves. ASICs calculate their processing power in the number of hash functions attempted per second. For this reason, crypto currencies "mines" (ASICs) are found in countries and regions around the globe where electricity is cheap [14]. These mining computers work virtually non-stop, trying to discover, as a hidden treasure, the future hash, that is, the mask of the entire chain of information that has ever existed across the network. The first ASIC to discover the hash of the future block - just like a miner who gives a golden nugget for the first time - will be rewarded with a virtual coin. Each node in the network carries a time stamp, which is a differentiator, i.e. it tells everyone if a computer has already found the valid solution (and generated a crypto-coin), because the rest of the computers involved in the same fever search to continue digging after the next, and the next, and so on. Each new block is chained to the previous one, being related to it through the results of the mathematical puzzle that the "miners" solve. This new block spreads over the network, notifying all participants of its existence. Once the notification is received, the other nodes undertake to resolve the next block. There are tens of thousands of knots in the world. Any node that has overcome the solution gets a block reward - a reward from the network that can be turned into traditional coins. To get a Bitcoin, it takes an average of 10 minutes. To obtain an Ethereum, the average time is between 10-12 seconds. If a computer resolves the equation sooner, the solution is considered too easy and the software will complicate the "problem" that needs to be solved to get the coin [15]. The world of cyber security is split. Some specialists say the blockchain is the safest way to protect any data. This is because there is no single command center and because each result can be verified in tens or thousands of sources, so it becomes impossible, for example, to insert into the system a fake Bitcoin. He will be identified immediately because it does not resemble the line from which he should come. All nodes in a blockchain network appear on a map that all "miners" have access to, after the computer's IP.

Among the most advanced and often used platforms for creating business software products, we can mention the following: EmcSSH, EmcSSL, Emc InfoCard, EmcTTS, Emc DPO, Emc Atom, Emc DNS. In terms of the EmcSSH platform, which is a further extension of SSH for network management, blockchain is a specialized repository of public keys (passwords) and user lists that have the right to authorized access. For example, a network user has access to multiple machines, a password-protected secret key file and a blockchain stored in the blocker, which excludes hacker attacks, is used to do this. If the impossible happens and the file is stolen, the user can immediately change it with another one or block the access. This method is most effective when managing a large number of servers, an ATM network, remote terminals, and so on. The most interesting thing is that this global control can be done regardless of geographic location with the same simplicity as in a regular local network. The EmcSSL platform is an extension of the custom SSL protocol. For him, the blockchain is the place where the so-called fingerprints of user certificates or individual organizations are stored. At the entrance, for example, on the bank's website and exchange of information between the user and Internet banking, user authentication takes place on the basis of such certificates, making it impossible to attempt the theft of passwords, codes or switches by third parties. When the certificate is lost, the restore order is sufficiently simplified, although it has some disadvantages in terms of imposed restrictions (the recovery service is a payment and you need some time to complete the procedure). The Emc InfoCard platform is based on an electronic business card system, inextricably linked to users' SSL certificates. Unlike the latter, these business cards are convenient because the information they contain can be changed. Such technology is indispensable in the legal field when it is necessary to prove the authenticity of a document, a contract, a patent, or even the publication of copyright. From the time of marking, you can easily check not only the date of issue or publication, but also the time to a second that in jurisprudence can 
sometimes play a key role. For example, two companies have signed a service contract. First, after signing the contract, he made the publication using TTS technology. The second company, unaware of this publication, attempts to make some changes to the contract over a period of time, motivating it to lose its copy, even maintaining that some changes were recorded in the first editorial of the deal. Here, thanks to the previous publication, it is easy to prove the correctness of the first company due to the temporary print imprint. The DPO system (EMA DPO) is another branch of the underlying technology used to prove the rights to any property in physical or intellectual terms. If we simplify it, the right to ownership of the car, house or apartment, land, software development, etc. can be confirmed at the basis of the unique numbers or features of such technologies. Similarly, it is possible to use inventory numbers, license plates, serial numbers, software licenses and in some cases (for real estate) - the location. Through this system, even re-registration of property rights can be done quite simply - it is just enough to do a data exchange operation in the blockchain. As will be clear, access to such operations can only be obtained by people who have the right to make this liqueur and any "outside" blend is excluded. Atom - a system for two-party transactions, without the involvement of a third party within the Emc Atom platform. In order to make it clear, in some cases, the transfer of property rights or the conclusion of contracts, the two parties must apply the services of notaries, banks or other organizations, whereas in our case their participation is no longer necessary. In the case of a transaction related to the property, the procedure of receipt of the money by the seller and the re-registration of ownership becomes a confirmation of the legality of the transactions with a preliminary check of the property to the seller and in the future confirmation of ownership of the new owner. However, the approach can only be used when both sides have unconditional mutual trust. Emc DNS is still a platform as an alternative domain name distribution system that prevents offenders who practice DNS attacks. With the use of this technology, any distributed network connected to the Internet becomes virtually invulnerable. Speed Transactions are a type of technology designed to deliver low-cost micropayments. Such calculations may have extremely small amounts. Since all operations are performed by the virtual system, it allows you to eliminate any speed limitation in the so-called TPS metric, not committing to the fact that each operation has its cost or expressed in cash equivalents or in consumed resources. It remains to be said that the advantages of the blockchain system are clearly expressed. The most important thing in technology is the perfect reliability and, as they say, the absolute safety. However, today there are active debates about the opportunity of widespread introduction of such technology. As a matter of fact, bankers use it slowly because of the low speed that initially depends on traffic, although they admit that performance has a lower priority compared to the reliability and security of operations. At the same time, any consumer using this technology can independently check the quality of the product purchased by tracking the entire merchandising and merchandising chain to ensure that the purchase meets the specified standards. However, it is still too early to talk about the worldwide introduction of this technology, which, according to many experts, is only comparable to the emergence of global Internet networks. The applications created on its basis, not to mention the organizations that use it, are not as many as we would like. However, most experts are willing to believe that the blockchain has a future related for example to assist information distribution for the Internet of Things [10].

\section{The impact of blockchain technology on mobile economy and platform examples}

Blockchain technology can change the operation of companies in the context of the new mobile economy, especially those who are currently confronted with a lack of transparency in the supply chain. When it comes to an extensive network, and we are confronted with such situations very often, it can be a real challenge to get a complete picture of the transactions made by suppliers, subcontractors and customers. The end-user may therefore have a problem in following the path of the individual elements that make up the final product. In view of increasing competition and the advancement of technical products, new technology is often needed. As far as transport and logistics are concerned, industrial standards for the use of blockchain technology have not yet been established, but this does not mean that nothing is happening. An American shipping company is testing a high-value tracking technology based on blockchain technology. We are convinced that this technology has a major impact on the supply chain, transport and logistics. This is a new frontier that will completely change the global supply chains. The first version of the VGM portal was released two years ago. The idea was to provide senders with a convenient solution for completing VGM (Verified Gross Mass) declarations that are required for maritime transport as a result of the SOLAS Convention. Today, with the help of blockchain technology, VGM serves about hundreds of thousands of monthly transactions, the VGM portal being permanently upgraded. All the information entered by the portal is stored in bulk, which allows the use of blockchain interfaces to exchange data with third parties so that no additional communication channels are needed. The list of promises related to the use of blockchain technology in the logistics sector is long, but its practical use is rare. The VGM portal allows you to acquire sufficient practical experience in using blockchain technology in many transactions in the domestic production environment. Technology brings benefits to customers by providing a secure solution for sharing information with third parties with greater efficiency and greater transparency. For example, this indicates involvement in a consortium that aims to digitize the shipping document for maritime freight transport. However, Fracht Group has begun cooperation with CargoX, 
a provider of innovative solutions based on the Smart Bill of Lading platform. The Fracht Group companies and their customers have gained the opportunity to use intelligent systems to manage maritime shipping documents, that is, billings. The CargoX platform allows companies to display and make available the original transport documents from the Ethereum blockchain network, facilitating the digital transfer of ownership of a shipping document and, therefore, the related assets from the sender to the recipient, the shipping agent, to the ship-owner or carrier. Ethereum is a cloud-based decentralized platform that manages smart contracts based on applications that work exactly as they are scheduled and without external interference. Accompanying documentation is made available in cloud encrypted form. A blockchain solution for managing and tracking container shipments. The first carrier, together with Maersk and IBM, is preparing a blockchainbased solution for managing and tracking container shipments, is a provider of integrated logistics solutions called Agility, that collect information on the management of individual shipments and then transfer this data through a decentralized and distributed transaction loghouse based on blockchain technology that is developed by IBM and Maersk. The goal is to reduce costs and improve the efficiency of operations, possibly through the integration of all transport information on a single secure platform at the expense of shippers, carriers, freight forwarders and other companies in the supply chain. The desired outcome is to improve efficiency and security in international trade. Blockchain technology reduces transport costs while increasing safety and reliability. Implementing it at an early stage, companies such as Agility can help Maersk and IBM understand the needs of shippers and create standards that increase the efficiency of commercial operations. A secure transaction log can be used to track shipments, create documentation, and record payments. Thanks to blockchain technology, Agility intends to develop supply chains in the most demanding regions around the world, offering customized solutions including air, sea and road transportation, storage, distribution, and help with specialized logistics projects, fairs and other events. In turn, the Infrastructure group, operating under Agility, can use the blockchain technology to manage industrial plants as well as to provide logistical support. According to estimates, the costs associated with document management and archiving are one-fifth of the \$ 1.8 billion spent annually on international freight transport. In the case of Maersk, they argue that the use of blockchain technology can not only facilitate the location of a particular container, but may also show the status of customs documents and transport documents. The technology can also be used by the customs and border authorities, providing the necessary information to carry out the risk analysis. This helps to increase safety and increase the efficiency of customs clearance and controls.

For now, only a tiny fraction of financial transactions are made with this technology, but the blockchain has managed to impress both large corporations and small entrepreneurs. Crypto currencies, such as Bitcoin, Ethereum, or Ripple, have not yet succeeded in replacing traditional payment methods, but the technology behind them still has the potential to bring about a technological revolution. It becomes more and more obvious that the banking system will be affected by the implementation of this technology, and its use may benefit large companies or small firms, state institutions, governments and especially people. Therefore, technology can be used to prevent financial fraud, voting fraud, reduce bank transaction costs, copyright, electronic signatures, document management, streams, concert tickets, online stores, accounting. Walmart Company is trying to use blockchain technology to facilitate the delivery, identification and verification of existing or marketed food products. If blockchain will be tested successfully within Walmart's retailer, this would be the world's largest deployment of technology to date. Walmart is the world's largest retailer and one of the largest companies in the world in general. Another application of blockchain technology is in the insurance industry. In this segment, technology has the potential to bring operational efficiency increase as well as to prevent fraud or improve the quality and safety of data change with third parties. For example, fraud can be prevented because anyone can check, from the police, authorities or those involved in the accident when an RCA insurance policy was issued. Fraud can be prevented because anyone can check, from the police, authorities or those involved in the accident when an RCA insurance policy was issued.

Another example of Blockchain's application is in the case of damages, which can be paid directly, without the need to open a claim file or to research and investigate the issue of insurance. In addition, the blockchain could also revolutionize the health system. Imagine how simple it is for a doctor to have all your health information without having to consult referrals, recipes, analysis results or other verbal information. The medical act would improve greatly, and the risk of malpractice would be diminished. Each patient is unique, with a particular evolution of a condition and a different response to a treatment. And in this context, it seems impossible to apply a common therapeutic strategy for all patients, even if they suffer from similar illnesses or even the same illnesses. Therefore, access to complete medical records is essential, so that the whole world can take a step forward towards what is called personalized medicine. As part of the medical records management, the blockchain can be used as a giant registry to allow the search and identification of all medical information related to the patient. Combating corruption and transparency in the allocation of public funds. There is another area in which blockchain technology could bring unimaginable success: spending public money. So if this is to be the case, technology can ensure transparent governance and the end of corruption. Blockchain technology is already taken into account by government representatives (US, UK) to combat corruption and rising public money. Thus, the Washington-based New America Task Force has proposed the use of blockchain-based applications to combat corruption. The idea now has a fairly substantial foundation 
after the initiative called the Blockchain. Trust Accelerator Initiative was launched. The Blockchain Trust Accelerator Initiative is a collaborative effort between New America, the BitFury blockfish company and the National Democratic Institute, through which partner entities plan to encourage collaboration with the government, private institutions and investors to develop and implement blockchain technologies for social good and a more efficient and rapid government. In the UK, it was proposed to use blockchain technology to track the use of State aid and grants for innovation and research. Thus, by making payments and registering grant funding, and how these funds are spent, the government will be able to prevent corruption by ensuring that money is not spent for purposes other than originally intended. The London Stock Exchange uses IBM's blockchain technology for small and medium-sized businesses, making them more transparent to attract investment. Food giants such as Nestle, Unilever or Walmart use technology in the supply and delivery system to increase food safety. In transport, Maersk and IBM are working on blockchain technology to track and monitor the global container ship's route. The Abu Dhabi National Bank uses the blockchain to offer crossborder transactions to its clients.

German car maker Mercedes-Benz has developed a blockchain platform. It looks like this huge company wants to keep up with that technology. The new Mercedes-Benz block-based platform will make the manufacturer's complex work processes more transparent and sustainable; and will help easier stock handling. ING Bank has bought a license for the use of a blockchain platform, valid for 5 years. The Dutch bank has concluded an agreement with the R3 consortium, specializing in block-chain technology. The agreement provides unlimited access to Corda Enterprise, the blockchain platform developed by R3. One of the main goals of the financial institution is to integrate CorDapp (the decentralized application on Corda) into its commercial infrastructure all over the world. According to Forbes, IBM launched at the end of 2018 the IBM Food Trust platform for global use across the food ecosystem. The company has tested the blockbased food tracing platform for more than 18 months. Project Coordinators said they would enroll on the platform for retailers, wholesalers and suppliers. Maqta Gateway, a wholly-owned subsidiary of Abu Dhabi Ports, has developed and launched its own blockchain solution for international logistics and commerce that will allow real-time tracking of merchandise containers and electronic documents, saving time and increasing the efficiency of international trade in a decentralized system. The blockchain solution has been developed to reduce the gap between exporters and importers in a recognized industry for aversion to digitization and change. It will achieve its goal by reducing the need to use paper-based documents, verbal communication and physical visits, interactions that will be replaced by rapid real-time information exchange through a decentralized registry.

Blockchain is basically a self-sustaining database that typically has a "wrapper functionality", an application development platform - or it can be seen as an operating system for which useful applications called smart Contracts. Smart contracts are programs that allow the transfer of value, money, documents, etc. in a perfectly transparent way, without any grounds for dispute and without the need for an intermediary, such as a bank, a lawyer, a notary, a clearing house and so on And they are still of great quality - not only are they defined all the rules that govern the contract, but these are automatically executed when the contractual terms agreed by the parties are fulfilled. Therefore, the source of the conflict caused by the eventual failure to contract is canceled, making this impossible. Ethereum, for example, an open-source project, was specifically created for creating smart contracts, which you can customize, but you have to pay for the computational power consumed with crypto Ethereum. Any application can be programmed, any concept can be abstracted, and Solidity is the programming language specifically created for development by Smart Contracts on Ethereum. Ether (ETH) is the virtual currency used in this ecosystem to pay for the deployment of intelligent contracts on the blockchain and mine (inclusion) in the Ethereum blockchain. Blockchain technology also allows distributed application development (DApps), which, like the blockchain protocol, can work on multiple computing units once, not on a single server. Therefore, we can say that all the computing units that make a blockchain work is a single computer, there is only one Ethereum computer in the world. And if the smart contract were the letters, distributed applications would be the words. OpenBasear is an eBay platform built on a blockchain that uses peer-to-peer payouts without any transaction fee. Blockchain has driven interest to another level, creating crowd-sourced venture capital funds - collective investment funds. With smart contracts, blockchain technology can allow voting systems to become transpa- tory, instant - anyone can see the results in real-time, there is no subjective and unalterable counting, votes do not gather in a server where they can be hacked. Any kind of collective decision can be taken immediately. The system would allow the vote at home - your identity in the blockchain is precise and unfathomable, which would drastically decrease absenteeism and would bring many millions to the ballot box. Aragon Core v0.5 is the most effective way to create and manage any type of decentralized organization. Aragon Network is the first community of decentralized organizations based on blockchain technology that aims to create cross-border digital law, a permission less system that protects the community from the interference of a state entity. Consumers can find out if what's on the label of a product is real or not. Shared repository technology allows once recorded information to be unavailable. Decentralizing data storage in the blockchain network brings clear benefits, protecting against any hacking activity. Smart contracts can protect intellectual property and, implicitly, sell online creative products, eliminating the risk of copying and redistributing them. Smart contracts can 
implement and ensure remote management of various IoT devices. There is a great need to manage personal data and person's identity on the web. The ability to verify a person's identity is the basis for all financial transactions on the internet. Blockchain offers secure methods to prove your identity, ensuring maximum security of this data. In the future, everyone will be able to personally manage their own data - including those generated by online activity - with the opportunity to sell them to research, research or research institutes that are interested in large data. Blockchain technology allows the creation of public records of document storage such as property documents, certificates of all kinds, diplomas, etc. - acts that are highly susceptible to being forged or stolen, doing so in a perfectly safe way and with a much higher cost and management efficiency than now. When running without intermediaries (banks, auditors, etc.), this exchange takes place almost instantly, compared to a few days of confirmation of the transaction, as is happening now.

\section{Conclusions}

As the number of devices able to connect to the Internet is getting bigger, the rapid increase in volume of data requires a bandwidth to measure. In 2020, the development of 5G-type networks will be accelerated, particularly for case studies in terms of value and uses. Networks will require new data traffic targeting mechanisms - edge-to-edge computing solutions as well as storage solutions. Already there is the concept of a smart home, but also a variety of distributors offering independent services. We expect these solutions, which can be applied to cities as well, will capture the interest of city administrations willing to implement the technology, but who now have difficulties when it comes to implementation. Cloud storage systems will become overloaded by the amount of existing data and will need to work in tandem with edge computing. Cloud storage will maintain scalability and processing power that can handle huge amounts of data. The computational edge system, being closer to the user, will provide the power of computing with the cost of reduced connectivity, but without the need for high security. We will see how blockchain becomes the core technology for decentralized systems. By providing a common trust-based work framework, blockchain will allow decentralized cooperation and coordination of disparate edge technology devices in a scalable way without a third party unnecessarily centralizing data. Deep learning is the equivalent of an enormous amount of data, experimental results and intense computational requirements. It is important to develop a culture of deep learning based on transparency and shared responsibility so that Deep0ps culture will expand rapidly, as it is the collaboration between data engineers and deep learning experts to build a deep learning network effective. In the field of payments, developers will work to ease consumer spending as much as possible. More and more non-banking companies will make their contribution to the evolution of payments, giving increasing priority to consumer experience. Healthcare IT companies will work to create a coherent system that includes patients, health care providers, and pharmacies. Only $20 \%$ of the industry's successful companies address the consumer directly. This trend will evolve, and experts believe that the remaining $80 \%$ can be differentiated by integrating regular workflows and industry regulations so that a coherent strategy will allow the health system to create more effective programs. According to the latest Cisco Visual Networking Index (VNI) report, more IP traffic will be generated in 2022 than in the 32 years since the launch of the Internet. By 2022, 60\% of the global population will use the internet, but new users will be greeted by a completely different Internet - one that connects people through portable devices, household appliances and automated vehicles. More than 28 billion devices and connections will be online, and more than $50 \%$ of them will be machine-to-machine (M2M). By 2022, video content will account for $82 \%$ of total IP traffic. The "live video" area is estimated to grow 15 times during 2017-2022 and will account for 17\% of all Internet video traffic. Also, VR traffic (virtual reality - VR) and AR (augmented reality - AR) will increase as more and more consumers and companies use these technologies.

The traffic associated with VR and AR applications will increase 12 times over the next five years. This year, we will see increased adoption of VR in business, from virtual demos and "test drives" to online product and real estate valuations. Mobile data traffic will continue to grow relative to other types of traffic in 2019. 3G and 4G will continue to be the most widely used protocols in 2019 as migration to $5 \mathrm{G}$ is still in its infancy. The Cisco VNI report estimates that by 2022, 22\% of global Internet traffic will come from mobile networks (from 12\% in 2017). Blockchain will continue to expand in areas such as supply chain management, networking, digital identity, and currency transactions. Most likely, all major cloud service providers will implement blockchain at the commercial level by the end of 2020, and this will become a key element in most $\mathrm{AI}$ and IoT offer over the next few years. More and more companies will need to focus on network transformation to continue delivering premium customer experiences. Currently networks have not been built to meet network needs in the coming years. Whether it's 5G, virtual reality, IoT or AI / ML, behind all these trends is a large-capacity digital network composed of a multitude of public and private networks. And because, by 2020 , it is estimated that one million items will be connected to the Internet every hour, this infrastructure is under great pressure. Manually managing networks becomes impossible. Companies must now be able to turn on any device, anywhere, at any time, regardless of the domain. The new architecture will have to be automated, anticipate actions, block security threats and continue to evolve and learn. Blockchains are secured by their design, being an example of a distributed computing system that has high cybertolerance tolerance. This makes blockchain technology suitable for recording events, medical records, and 
recording other management activities such as identity management, transaction processing, provenance, tracking the food trade route, or voting and financing systems various projects.

\section{References}

1. Augot, D., Chabanne, H., Chenevier, T., George, W., Lambert, L. (2017): A user-centric system for verified identities on the bitcoin blockchain. In: Garcia-Alfaro, J., Navarro-Arribas, G.,Hartenstein, H., Herrera-Joancomartí, J. (eds.) ESORICS/DPM/CBT -2017. LNCS, vol.10436, pp. 390-407. Springer, Cham. https://doi.org/10.1007/978-3-319-67816$\underline{0.22}$

2. Bhardwaj, S., Kaushik, M. (2018): Blockchain-technology to drive the future. In: Satapathy, S.C., Bhateja, V., Das, S. (eds.) Smart Computing and Informatics. SIST, vol. 78, pp. 263-271. Springer, Singapore. https://doi.org/10.1007/9783-319-59427-9 76

3. Castellanos, J., Coll-Mayor, D., Notholt, J. (2017): Cryptocurrency as guarantees of origin: simulating a green certificate market with the Ethereum Blockchain. In: 2017 IEEE, International Conference on Smart Energy Grid Engineering (SEGE)

4. Grumbach, S., Riemann, R. (2017): Distributed random process for a large-scale peer-to-peer lottery. In: Chen, L.Y., Reiser, H.P. (eds.) DAIS 2017. LNCS, vol. 10320, pp. 34-48. Springer, Cham. https://doi.org/ 10.1007/978-3-319-59665$5 \_3$

5. Liu, P.T.S. (2016): Medical record system using blockchain, big data and tokenization. In: Lam, K.-Y., Chi, C.-H., Qing, S. (eds.) ICICS 2016. LNCS, vol. 9977, pp. 254-261. Springer, Cham (2016). https://doi.org/10.1007/978-3-319-50011-9_20

6. Mengelkamp, E., Notheisen, B., Beer, C., Dauer, D., Weinhardt, C. (2017): A blockchain-based smart grid: towards sustainable local energy markets. Comput. Sci. Res. Dev. 33, 207-214. https://doi.org/10.1007/s00450-017-0360-9

7. Munsing, E., Mather, J., Moura, S. (2017): Blockchains for decentralized optimization of energy resources in microgrid networks. In:IEEE Conference on Control Technology and Applications (CCTA) (2017). https://doi.org/10.1109/ CCTA.2017.8062773

8. O'Leary, K., O’Reilly, P., Feller, J., Gleasure, R., Li, S., Cristoforo, J. (2017): Exploring the application of blockchain technology to combat the effects of social loafing in cross functional group projects. In: Proceedings of the 13th International Symposium on Open Collaboration - OpenSym2017. https://doi.org/ 10.1145/3125433.3125464

9. Probst, L., Frideres, L., Cambier, B., Martinez-Diaz, C.: PwC Luxemburg (2016): Blockchain Applications and Services, Directorate-General for Internal Market, Industry, Entrepreneurship and SMEs, European Union

10. Polyzos, G., Fotiou, N. (2017): Blockchain-assisted information distribution for the Internet of Things. In IEEE International Conference on Information Reuse and Integration (IRI)

11. Suhaliana bt Abd Halim, N., Rahman, M.A., Azad, S., Kabir, M.N. (2018): Blockchain security hole: issues and solutions. In: Saeed, F., Gazem, N., Patnaik, S., Saed Balaid, A.S., Mohammed, F. (eds.) IRICT 2017. LNDECT, vol. 5, pp. $739-746$. Springer, Cham. https://doi.org/10.1007/978-3-319-59427-9_76

12. Steger, M., Dorri, A., Kanhere, S., Römer, K., Jurdak, R., Karner, M. (2017): Secure Wireless Automotive Software Updates Using Blockchains: a proof of concept. Adv. Microsyst. Autom. Appl. 2017, 137-149

13. Vo, H., Mehedy, L., Mohania, M., Abebe, E. (2017): Blockchain-based data management and analytics for micro-insurance applications. In: Proceedings of the 2017 ACM on Conference on Information and Knowledge Management - CIKM 2017

14. Tsai, W., Blower, R., Zhu, Y., Yu, L. (2016): A system view of financial blockchains. In: 2016 IEEE Symposium on ServiceOriented System Engineering (SOSE)

15. Zhao, Z., Chan, T.-H.H. (2015): How to vote privately using bitcoin. In: Qing, S., Okamoto, E., Kim, K., Liu, D. (eds.) ICICS 2015. LNCS, vol. 9543, pp. 82-96. Springer, Cham (2016). https://doi.org/10.1007/978-3-319-29814-6_8

16. Zhao, H., Zhang, Y., Peng, Y., Xu, R. (2017): Lightweight backup and efficient recovery scheme for health blockchain keys. In IEEE 13th International Symposium on Autonomous Decentralized System (ISADS). https://doi.org/10.1109/ ISADS.2017.22

17. Zhao, J., Fan, S., Yan, J. (2016): Overview of business innovations and research opportunities in blockchain and introduction to the special issue. Finan. Innov. 2

18. Xu, Y., Li, Q., Min, X., Cui, L., Xiao, Z., Kong, L. (2016): E-commerce blockchain consensus mechanism for supporting highthroughput and real-time transaction. In: Wang, S., Zhou, A. (eds.) CollaborateCom. LNICST, vol. 201, pp. 490-496. Springer, Cham (2017). https://doi.org/10.1007/978-3-319-59288-6_46

19. Zibin Zheng1, Shaoan Xie1, Hongning Dai2, Xiangping Chen4, and Huaimin W., (2018): An Overview of Blockchain Technology: Architecture, Consensus, and Future Trends. IEEE 6th International Congress on Big Data

20. Wijaya, D.A., Liu, J.K., Suwarsono, D.A., Zhang, P. (2017): A new blockchain-based value-added tax system. In: Okamoto, T., Yu, Y., Au, M.H., Li, Y. (eds.) ProvSec 2017. LNCS, vol. 10592, pp. 471-486. Springer, Cham. https://doi.org/10.1007/978-3-319-68637-0_28 